\title{
KECK SPECTROSCOPY OF FAINT $3<z<8$ LYMAN BREAK GALAXIES: EVIDENCE FOR A DECLINING FRACTION OF EMISSION LINE SOURCES IN THE REDSHIFT RANGE $6<z<8$
}

\author{
Matthew A. Schenker ${ }^{1}$, Daniel P. Stark ${ }^{2}$, Richard S. Ellis ${ }^{1}$, Brant E. Robertson ${ }^{1}$, James S. Dunlop ${ }^{3}$, \\ Ross J. McLure ${ }^{3}$, Jean-Paul KneiB ${ }^{4}$, And Johan Richard ${ }^{5}$ \\ ${ }^{1}$ Department of Astrophysics, California Institute of Technology, MS 105-24, Pasadena, CA 91125, USA \\ ${ }^{2}$ Kavli Institute of Cosmology \& Institute of Astronomy, University of Cambridge, Madingley Road, Cambridge CB3 0HA, UK \\ ${ }^{3}$ Institute for Astronomy, University of Edinburgh, Royal Observatory, Edinburgh EH9 3HJ, UK \\ ${ }^{4}$ Laboratoire d'Astrophysique de Marseille, F-13388 Marseille cedex 13, France \\ ${ }^{5}$ Observatoire de Lyon, F-69561 Saint-Genis-Laval, France \\ Received 2011 July 7; accepted 2011 September 29; published 2011 December 22
}

\begin{abstract}
Using deep Keck spectroscopy of Lyman break galaxies selected from infrared imaging data taken with the Wide Field Camera 3 on board the Hubble Space Telescope, we present new evidence for a reversal in the redshift-dependent fraction of star-forming galaxies with detectable Lyman alpha (Ly $\alpha)$ emission in the redshift range $6.3<z<8.8$. Our earlier surveys with the DEIMOS spectrograph demonstrated a significant increase with redshift in the fraction of line emitting galaxies over the interval $4<z<6$, particularly for intrinsically faint systems which dominate the luminosity density. Using the longer wavelength sensitivities of Low Resolution Imaging Spectrometer and NIRSPEC, we have targeted 19 Lyman break galaxies selected using recent WFC3/IR data whose photometric redshifts are in the range $6.3<z<8.8$ and which span a wide range of intrinsic luminosities. Our spectroscopic exposures typically reach a $5 \sigma$ sensitivity of $<50 \AA$ for the rest-frame equivalent width (EW) of Ly $\alpha$ emission. Despite the high fraction of emitters seen only a few hundred million years later, we find only two convincing and one possible line emitter in our more distant sample. Combining with published data on a further seven sources obtained using FORS2 on the ESO Very Large Telescope, and assuming continuity in the trends found at lower redshift, we discuss the significance of this apparent reversal in the redshift-dependent Ly $\alpha$ fraction in the context of our range in continuum luminosity. Assuming all the targeted sources are at their photometric redshift and our assumptions about the Ly $\alpha$ EW distribution are correct, we would expect to find so few emitters in less than $1 \%$ of the realizations drawn from our lower redshift samples. Our new results provide further support for the suggestion that, at the redshifts now being probed spectroscopically, we are entering the era where the intergalactic medium is partially neutral. With the arrival of more sensitive multi-slit infrared spectrographs, the prospects for improving the statistical validity of this result are promising.
\end{abstract}

Key words: galaxies: evolution - galaxies: formation - galaxies: high-redshift - galaxies: starburst

Online-only material: color figures

\section{INTRODUCTION}

Determining when neutral hydrogen in the intergalactic medium (IGM) was reionized is an important question in observational cosmology and a precursor to understanding whether star-forming galaxies provided the necessary UV photons (Robertson et al. 2010). One of the most practical probes of reionization with current facilities utilizes the frequency of occurrence of Ly $\alpha$ emission in star-forming galaxies. As Ly $\alpha$ photons are resonantly scattered by neutral hydrogen, the abundance of Ly $\alpha$ emitters (LAEs) should decrease as observations probe into the era where there is neutral gas (e.g., Malhotra \& Rhoads 2004; Ouchi et al. 2010; Kashikawa et al. 2011). The recent discovery of large numbers of candidate galaxies beyond $z \simeq 7$ through multi-color imaging undertaken with the infrared Wide Field Camera (WFC3/IR) on board Hubble Space Telescope (HST; e.g., Bouwens et al. 2010b; Bunker et al. 2010; Finkelstein et al. 2010; McLure et al. 2010) now makes it feasible to track the occurrence of $\operatorname{Ly} \alpha$ line emission to interesting redshifts where neutral hydrogen may be present.

Of course, astrophysical factors other than a neutral IGM can also affect the presence of $\operatorname{Ly} \alpha$ emission. Because of this, an alternative approach for gauging when reionization occurred, introduced by Stark et al. (2010, hereafter Paper I), is to spectroscopically measure the fraction of Lya emission within color-selected Lyman break galaxy (LBG) populations. Tracking the redshift-dependent fraction in a well-defined population avoids consideration of absolute changes in the host galaxy number density such as has been the case in studies based on the luminosity function of narrowband-selected LAEs. Furthermore, evolution in dust obscuration can be independently tracked via correlations seen with the colors of the rest-frame UV continuum. Paper I presented a comprehensive survey of over 600 LBGs with deep spectra, mostly undertaken with the DEIMOS instrument on Keck (Faber et al. 2003), but including published samples from the Very Large Telescope (VLT; Vanzella et al. 2009 and references therein). In that paper we demonstrated the utility of the method and discussed the paucity of line emission in gravitationally lensed $z>7$ candidates from the sample of Richard et al. (2008).

In Stark et al. (2011, hereafter Paper II), through ultra-deep exposures with DEIMOS we significantly improved the line emission statistics at $z \simeq 6$, providing a robust measure of the rest-frame equivalent width (EW) distribution of Ly $\alpha$ emission at the highest redshift when the universe is believed to be fully ionized (Fan et al. 2006; cf. Mesinger 2010). This provides a sound basis for predicting the likelihood of emission at higher redshift and thereby enabling a test of whether there is absorption by neutral gas. Significantly, we found that over $50 \%$ of moderately faint $\left(-20.25<M_{\mathrm{UV}}<-18.75\right) z \simeq 6$ 
LBGs exhibit strong emission with rest-frame EWs $>25 \AA$. As this fraction increases over $4<z<6$, we argued on continuity grounds that we should expect a high success rate in recovering line emission from the newly found WFC3/IR samples of $z>7$ LBGs unless we encounter a more neutral IGM in the short time interval prior to $z \simeq 7$. Some evidence for this is seen in the recent studies of Fontana et al. (2010) and Vanzella et al. (2011).

The present paper is concerned with an initial application of this test to the newly available sample of WFC3/IR candidates with photometric redshifts in the redshift range $6.3<z<8.8$. Two important factors have motivated and shaped our program. First, it is important to note that $\operatorname{Ly} \alpha$ emission is the only spectroscopic redshift indicator for galaxies beyond $z \simeq 6$. Since it is the absence of strong Ly $\alpha$ emission that provides the basis for considering an increased neutral fraction, it is important to be sure that the targets are truly at the expected redshifts. Many early candidate LBGs believed to lie beyond $z \simeq 6-7$ remained controversial because of their limited or marginal photometry. The improved filter set and superior performance of WFC3/IR has given us confidence that the current list of $z \simeq 7-8$ candidates is more robust than those based on earlier NICMOS data (Robertson et al. 2010). Second, to match the lower redshift data, sampling from a similarly wide range of LBG luminosities, as we do here, will be advantageous. As shown in Papers I and II, the fraction of line emission increases in intrinsically fainter sources and so by comparing fractions with respect to their LBG luminosities, we may gain additional evidence for the onset of the neutral era.

Throughout the paper, we adopt a $\Lambda$-dominated, flat universe with $\Omega_{\Lambda}=0.7, \Omega_{M}=0.3$, and $H_{0}=70 h_{70} \mathrm{~km} \mathrm{~s}^{-1} \mathrm{Mpc}^{-1}$. All magnitudes in this paper are quoted in the AB system (Oke \& Gunn 1983).

\section{OBSERVATIONS}

In compiling a target list for this program, we are guided by the need for a robust photometric redshift for each galaxy based on improved photometry from WFC3/IR and a range of rest-frame UV luminosities $\left(M_{\mathrm{UV}}{ }^{6}\right)$. Our primary source of targets for the wide-field multi-slit capabilities of the Low Resolution Imaging Spectrometer (LRIS) on the Keck I telescope (Oke et al. 1995) equipped with a new red-sensitive CCD was $i^{\prime}-$ and $z^{\prime}$-drop candidates whose photometric redshifts $z>6.3$ from the HST Early Release Science (ERS) field (Hathi et al. 2010; McLure et al. 2011). The grating for these observations was blazed at 600 lines $\mathrm{mm}^{-1}$. On 2011 January 7 and February 4 we secured $7 \mathrm{hr}$ of on-source integration for eight suitable targets on a single mask using slit widths of $1^{\prime \prime}$, observed through a median seeing of 0.98 .

In a more ambitious campaign probing to higher redshift we also targeted three $z^{\prime}$-drop sources from the Hubble Ultradeep Field (HUDF) P34 field (GO 11563, PI: Illingworth) and an additional gravitationally lensed source in the cluster MS0451-03 (GO 11591, PI: Kneib) using the near-infrared spectrograph NIRSPEC (McLean et al. 1998) during 2010 November 14-17 and 2011 January 14-15. This extends our search for $\mathrm{Ly} \alpha$ emission up to a redshift $z \simeq 8.2$. Although we undertook extended integrations on all four sources with a 0.76 slit, tracking difficulties affected some exposures. To determine the effective on-source integration time, we secured our astrometric position for each exposure by locating objects visible in the slit viewing camera to a precision of $\sim 0^{\prime} .2$. We continued

\footnotetext{
6 Corresponding to a rest wavelength $\lambda \simeq 1500 \AA$ (Paper I).
}

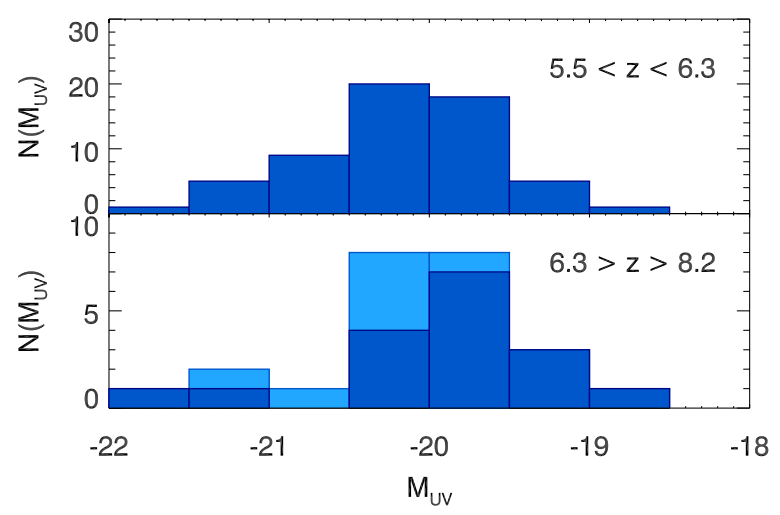

Figure 1. Distribution of rest-frame UV absolute magnitudes, $M_{\mathrm{UV}}$, for $i^{\prime}$-drop sources discussed in Paper II with $z \sim 5.5-6.3$ (top panel) compared with those for the present survey of $i^{\prime}$ - and $z^{\prime}$-drops at $z \geqslant 6.3$ (lower panel). There is an additional dropout, A2261_1, not shown on this histogram at $M_{\mathrm{UV}} \simeq-16$. Dark shading in the lower panel refers to sources selected on the basis of WFC3/IR imaging in the Keck campaign, (Table 1); light shading refers to additional data drawn from the VLT campaign of Fontana et al. (2010).

(A color version of this figure is available in the online journal.)

this campaign over 2011 May 15-18. During these four nights, we did not encounter any tracking difficulties and, in excellent conditions, successfully used NIRSPEC to study an additional seven WFC3-IR dropouts drawn from numerous surveys: the BoRG pure parallel survey (Trenti et al. 2011; also independently discovered by Yan et al. 2011), the Extended Groth Strip (EGS) region in CANDELS (Grogin et al. 2011; Koekemoer et al. 2011), and the lensing clusters A1703 (GO 10325, PI: Ford; Bradley et al. 2011) and A2261 (CLASH survey; Postman et al. 2011).

We reduce the LRIS data following standard procedures, with bias subtraction and flat-fielding using dome exposures. We used the Kelson (2003) code to remove spatial and spectral distortion and to model and subtract the sky emission. Wavelength calibration was determined directly from sky lines. A final two-dimensional spectrum was extracted for each object with pixels binned $\operatorname{logarithmically}$ by $\Delta \log (\lambda)=4.02 \times 10^{-5}$. As in Papers I and II, we search through the two-dimensional spectrum visually to identify emission lines, and confirm these with a boxcar extracted one-dimensional spectrum.

Our exposures with NIRSPEC were conducted with typical spatial dithering of $5^{\prime \prime}$. In the case of some lensed sources, we dithered by longer amounts to ensure the arc was oriented along the slit, and was not spatially coincident with any background objects. We flat-fielded and sky-subtracted the spectra using IDL routines written by G. Becker (2010, private communication). To compute the camera distortion and spectral curvature, we fit traces of standard stars along the slit, and skylines perpendicular to the slit. From this, we then derive a wavelength and sky position for all pixels in each two-dimensional spectrum, which were used to align the individual exposures. A final spectrum was created for each object by median stacking all exposures to eliminate signals from cosmic ray strikes.

In total, this paper therefore presents the results of Keck spectroscopy for 19 WFC3-IR selected sources whose photometric redshifts lie in the range $6.3<z<8.8$. A summary of the new observations is given in Table 1. To this sample, we add a further seven $z>6.3$ sources discussed by Fontana et al. (2010). Figure 1 compares the UV absolute magnitude distribution of the combined sample with that presented for the redshift range $z \simeq 5-6$ in Paper II; clearly the samples span a similar 
Table 1

Catalog of Sources with Keck Spectroscopy and Emission Line Properties, when Detected

\begin{tabular}{|c|c|c|c|c|c|c|c|c|c|c|}
\hline ID & R.A. & Decl. & $z 850$ & $J_{125}$ & $H_{160}$ & $\mu^{\mathrm{a}}$ & $\begin{array}{c}z_{\text {phot }} \\
\text { (hr) }\end{array}$ & $t_{\exp }$ & $z$ & $\begin{array}{l}\text { EW } \\
(\AA)\end{array}$ \\
\hline \multicolumn{11}{|c|}{ LRIS } \\
\hline ERS $5847^{\mathrm{b}, \mathrm{c}}$ & $03: 32: 16.0$ & $-27: 43: 01.4$ & $26.6(0.1)^{\mathrm{d}}$ & $26.6(0.1)$ & $26.7(0.1)$ & $\ldots$ & 6.48 & 7 & & \\
\hline ERS $7376^{b, c}$ & $03: 32: 29.5$ & $-27: 42: 04.5$ & $27.2(0.1)^{\mathrm{d}}$ & $27.0(0.1)$ & $27.0(0.1)$ & $\ldots$ & 6.79 & 7 & & \\
\hline ERS 7412 & $03: 32: 10.0$ & $-27: 43: 24.0$ & $26.9(0.1)^{\mathrm{d}}$ & $27.0(0.1)$ & $26.7(0.1)$ & $\ldots$ & 6.38 & 7 & & \\
\hline ERS 8119 & $03: 32: 29.5$ & $-27: 41: 32.7$ & $27.7(0.2)^{\mathrm{d}}$ & $27.1(0.1)$ & $27.5(0.1)$ & $\ldots$ & 6.78 & 7 & & \\
\hline ERS 8290 & $03: 32: 13.4$ & $-27: 42: 30.9$ & $27.3(0.1)^{\mathrm{d}}$ & $27.1(0.1)$ & $26.8(0.1)$ & $\ldots$ & 6.52 & 7 & & \\
\hline ERS $8496^{\mathrm{d}}$ & $03: 32: 29.7$ & $-27: 40: 49.9$ & $27.2(0.1)^{\mathrm{d}}$ & $27.3(0.1)$ & $27.5(0.1)$ & $\ldots$ & 6.52 & 7 & 6.441 & $69 \pm 10$ \\
\hline ERS 10270 & $03: 32: 29.5$ & $-27: 42: 54.0$ & $28.1(0.1)^{\mathrm{d}}$ & $27.4(0.1)$ & $28.0(0.2)$ & $\ldots$ & 7.02 & 7 & & \\
\hline ERS 10373 & 03:32:27.0 & $-27: 41: 42.9$ & $27.5(0.1)^{\mathrm{d}}$ & $27.4(0.1)$ & $27.8(0.2)$ & $\ldots$ & 6.44 & 7 & & \\
\hline \multicolumn{11}{|c|}{ NIRSPEC } \\
\hline $\mathrm{A} 1703 \_z D 1^{\mathrm{e}}$ & $13: 14: 59.4$ & 51:50:00.8 & $25.8(0.2)$ & $24.1(0.1)$ & $24.0(0.1)$ & 9.0 & 6.75 & $2, \ldots$ & & \\
\hline A1703_zD3 ${ }^{\mathrm{e}}$ & $13: 15: 06.5$ & $51: 49: 18.0$ & $26.8(0.5)$ & $25.5(0.1)$ & $25.1(0.2)$ & 7.3 & 6.89 & $2, \ldots$ & & \\
\hline A1703_zD6 ${ }^{\mathrm{e}}$ & $13: 15: 01.0$ & 51:50:04.3 & $27.9(0.5)$ & $25.8(0.1)$ & $25.9(0.1)$ & 5.2 & 7.02 & 5,3 & 7.045 & $65 \pm 12$ \\
\hline $\mathrm{A} 1703 \_z D 7^{\mathrm{e}}$ & $13: 15: 01.3$ & $51: 50: 06.1$ & $>28.5$ & $26.8(0.2)$ & $26.4(0.2)$ & 5.0 & 8.80 & 5,3 & & \\
\hline A2261_1 & $17: 22: 28.7$ & $32: 08: 30.9$ & $>28.6$ & $26.9(0.1)$ & $27.3(0.1)$ & 3.5 & 7.81 & $5.7, \ldots$ & & \\
\hline BoRG_58_1787_1420 f & $14: 36: 50.6$ & $50: 43: 33.6$ & $>27.9^{\mathrm{d}}$ & $25.8(0.1)$ & $25.9(0.2)$ & $\cdots$ & 8.27 & 2,3 & & \\
\hline EGS_K1 & $14: 19: 24.2$ & $52: 46: 36.2$ & $>27.8^{g}$ & $25.3(0.1)$ & $25.4(0.1)$ & $\ldots$ & 8.27 & $2.5, \ldots$ & & \\
\hline HUDF09_799h & 03:33:09.1 & $-27: 51: 55.4$ & $>29.1$ & $27.7(0.1)$ & $27.6(0.2)$ & $\cdots$ & 6.88 & $4.5, \ldots$ & & \\
\hline HUDF09_1584 ${ }^{\mathrm{h}}$ & $03: 33: 03.8$ & $-27: 51: 20.4$ & $27.2(0.1)$ & $26.7(0.1)$ & $26.6(0.1)$ & $\cdots$ & 7.17 & $5.5, \ldots$ & & \\
\hline HUDF09_1596 & $03: 33: 03.8$ & $-27: 51: 19.6$ & $27.3(0.1)$ & $26.8(0.1)$ & $26.8(0.1)$ & $\ldots$ & 7.45 & $5.5, \ldots$ & $6.905 ?$ & $30 \pm 15$ \\
\hline MS0451-03_10 & 04:54:08.8 & $-3: 00: 29.1$ & $>28.3$ & $26.7(0.1)$ & $26.9(0.1)$ & 50 & 7.50 & $2.5, \ldots$ & & \\
\hline
\end{tabular}

Notes. $1 \sigma$ magnitude errors are listed in parenthesis. For non-detections, we list $2 \sigma$ limiting magnitudes. For our NIRSPEC targets, times correspond to exposures in filters N1 and N2, which cover wavelength ranges of 9470-11210 $\mathrm{A}$ and 10890-12930 A, respectively.

${ }^{a}$ Best-fit magnification for sources in our sample which are gravitationally lensed.

b In order listed in table, discovered as Objects 1,4,3 in Wilkins et al. (2010).

${ }^{c}$ In order listed in table, identified as ERS.z.45856 and ERS.z.87209 in Wilkins et al. (2011).

d $Y_{098 M}$.

e Discovered in Bradley et al. (2011).

${ }^{\mathrm{f}}$ Discovered in Trenti et al. (2011).

g $I_{814 \mathrm{~W}}$.

${ }^{\mathrm{h}}$ In order listed in table, discovered as P34.z.6106 and P34.z.4809 in Wilkins et al. (2011).

luminosity range. This luminosity range is broader than the recent work of Ono et al. (2011) and Pentericci et al. (2011). In similar spectroscopic follow-up campaigns, they target brighter dropouts, primarily with $M_{\mathrm{UV}}<-21.4$, and $-21.75<$ $M_{\mathrm{UV}}<-20.0$, respectively.

Remarkably, from the new Keck exposures, we find very few convincing detections of line emission. Figure 2 (bottom panel) shows a two-dimensional spectroscopic montage of four sources for which line emission may be present, of which one case (HUDF09_1596) is marginal $(2 \sigma)$ and the other (ERS 8290) lies outside the expected redshift range if it is Ly $\alpha$.

The emission line apparently seen in ERS 8290 (a $z^{\prime}$-band dropout) is detected at $>5 \sigma$ with a flux of $6.7 \pm 0.8 \times$ $10^{-18} \mathrm{erg} \mathrm{cm}^{-2} \mathrm{~s}^{-1}$ at $\lambda 7644 \pm 2 \AA$. It also exhibits the asymmetric profile characteristic of Ly $\alpha$ in the one-dimensional extraction, but this would place the object at $z=5.286$, quite discrepant from our photometric estimate of $z=6.52$. However, upon examining the positioning of our LRIS slits more carefully (bottom panel of Figure 2), we find there is a faint $V$-drop candidate with $i^{\prime}=27.5$ and $z^{\prime}=27.9$ only $0^{\prime \prime} .4$ away which would have been at least partially visible through our slit during the exposure. After subtracting the line flux from the $i^{\prime}$-band photometry, we determine a photometric redshift of $z=4.91$, in reasonable agreement with the Keck spectroscopy, particularly given a greater line flux (as is likely given the object's poor positioning in the slit) would increase the photometric redshift estimate. The resolution of the confusion arising from these two proximate sources emphasizes again the prominence of Ly $\alpha$ emission in low-luminosity $z \simeq 5-6$ sources (Papers I and II).

The two satisfactory detections refer to emission at $\lambda 9045 \pm 2 \AA$ for ERS 8496 in the LRIS mask and emission at $\lambda 9780 \pm 4 \AA$ in the NIRSPEC exposure of the lensed source A1703_zD6. Both objects are detected at $\geqslant 5 \sigma$ in our final exposures. In our one-dimensional extraction of ERS 8496, the emission line has an FWHM of $9 \pm 1 \AA$, and displays an asymmetric profile with a steeply rising blue edge and slowly decaying red tail, characteristic features of Ly $\alpha$ at high redshift. Because our spectral resolution is significantly lower (6.5 $\AA$ for NIRSPEC versus $4.1 \AA$ for LRIS) in our spectrum of A1703_zD6, we are unable to determine any line profile information. The emission feature is seen independently in coadditions on two separate nights, indicating its reality.

If both lines are Ly $\alpha$, the implied spectroscopic redshifts for ERS 8496 and A1703_zD6 are $z=6.441 \pm 0.002$ and $7.045 \pm 0.004$, respectively, in excellent agreement with our photometric predictions of 6.52 and that of 7.0 derived by Bradley et al. (2011). The measured line fluxes for the two objects are $9.1 \pm 1.4$ and $28.4 \pm 5.3 \times 10^{-18} \mathrm{erg} \mathrm{cm}^{-2} \mathrm{~s}^{-1}$. We then assume a spectral slope of $\beta=-2$, which is characteristic of galaxies at this redshift (Dunlop et al. 2011), though there may be evidence for steeper slopes at sub- $L_{\star}$ luminosities (Bouwens et al. 2010a). Under this assumption, taking the magnitude from the first filter in which the object is detected $\left(Y_{098 M}\right.$ for ERS 8496 , and $J_{125}$ for 1703 zD6), we calculate EWs of $69 \pm 10$ 

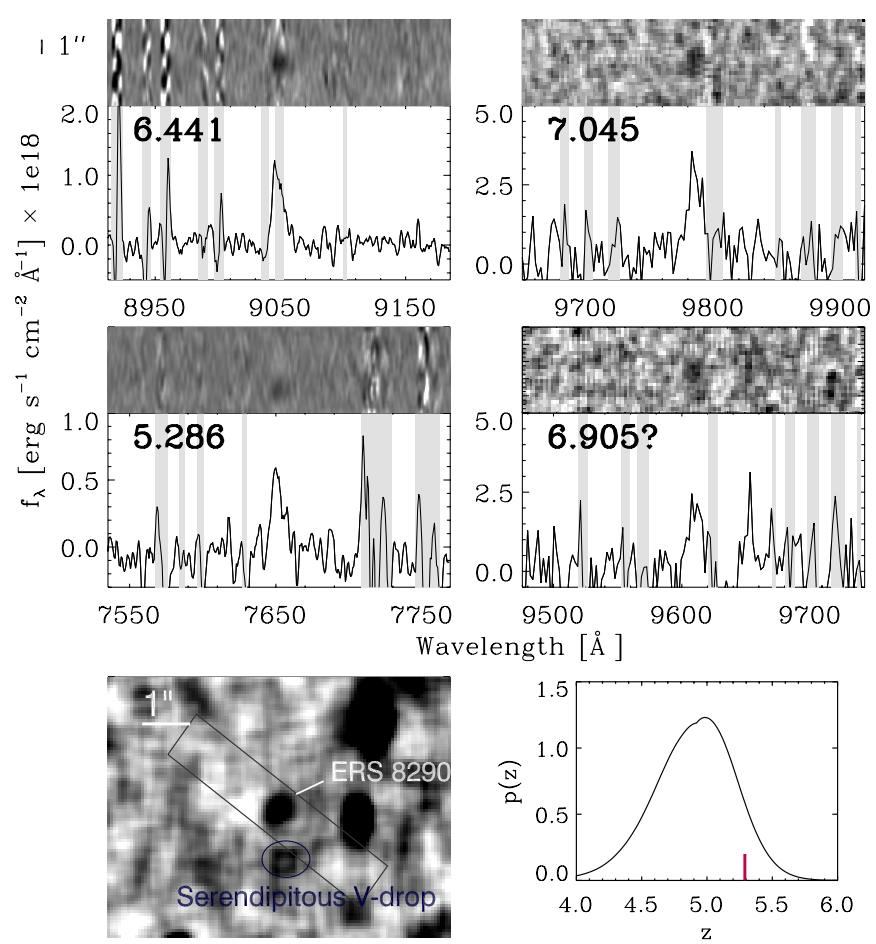

Figure 2. Montage of Ly $\alpha$ emission detected from four sources in our Keck survey, along with boxcar-extracted one-dimensional spectrum. Wavelength ranges contaminated by strong skylines are shaded in gray in the onedimensional extraction. The top row shows the two robust detections of ERS 8496 and A1703_zD6 at $z=6.441$ and $z=7.045$, respectively. The bottom row shows a marginal detection at $z=6.905$ for HUDF09_1596 and a likely Ly $\alpha$ line at $z=5.286$ arising from a serendipitous $V$-drop close to ERS 8290 as illustrated in the bottom left slit image. The bottom right panel shows the photometric redshift distribution for this serendipitous $V$-drop, with a vertical line indicating the observed spectroscopic redshift (see the text for further details).

and $65 \pm 12 \AA$, respectively. Because our objects have additional coverage longward of the detection filter, we can also compute a value for $\beta$, and extrapolate to find the continuum flux at $\lambda_{\text {rest }}=1216 \AA$. Using the formulae of Dunlop et al. (2011), we find $\beta=-2.39 \pm 0.55$, and $-2.44 \pm 0.64$. When computing EWs using this method, we obtain $67 \pm 11$ and $59 \pm 12 \AA$, respectively.

\section{ANALYSIS}

Our approach in this paper is to compare the rate of occurrence of Ly $\alpha$ in our new $6.3<z<8.8$ sample with that expected from our reference sample of $i^{\prime}$-drops with $5.5<z<6.3$ drawn from Paper II (see Figure 1). In both Papers I and II we showed that the rest-frame EW distribution is a function of restframe UV absolute magnitude, $M_{\mathrm{UV}}$, and thus we additionally take this luminosity dependence into account. We estimate the luminosities of all our sources in Table 1 from their photometric redshift and incorporate the lensing magnification $\mu$ for our lensed sources from Bradley et al. (2011) for A1703 and from the mass model of Richard et al. (2010) for MS0451-03 and A2261. For our baseline Ly $\alpha$ EW distribution, we use the data from of Paper II at $z \simeq 6$, separated into high- and low-luminosity regimes.

The fraction of emitters within each bin of EW $>25 \AA$ is taken directly from Figure 2 of Paper II. We set the slope of the distribution within an EW bin equal to the slope between the two lowest bins in Paper II, $25 \AA<$ EW $<55 \AA$, and
$55 \AA<\mathrm{EW}<85 \AA$. This slope is equal to $d p(\mathrm{EW}) / d \mathrm{EW}=$ -0.0030 for the lower luminosity sample $\left(-20.25<M_{\mathrm{UV}}<\right.$ $-18.75)$, and -0.0017 for the higher luminosity sample $\left(-21.75<M_{\mathrm{UV}}<-20.25\right)$. To create the probability distribution for galaxies with EW less than $25 \AA$, we extrapolate to $\mathrm{EW}=0 \AA$ using this slope, and assign the remaining fraction of galaxies as non-emitters. In Papers I and II we also showed the fraction of emitters is a function of redshift, rising significantly for lower luminosity sources over $4<z<6$, most likely as a result of reduced dust extinction in the early Lyman break population. Therefore, as discussed in Paper II, we have also used a projected rest-frame EW distribution at $z \simeq 7$ assuming this evolutionary trend continues beyond $z \simeq 6$.

Two key factors enter into the calculation of the visibility of line emission in a ground-based survey. First, for any target with a particular photometric redshift likelihood function $p(z)$, it may be that the spectral region surveyed by LRIS or NIRSPEC does not completely cover the expected wavelength range where Ly $\alpha$ might be present. Second, the EW limit for Ly $\alpha$ emission will be a highly non-uniform function of wavelength due to the mitigating effect of night-sky emission. Provided the photometric redshift solution we derive is robust, we can estimate both factors and hence derive the likelihood of seeing Ly $\alpha$ for each of our 26 sources assuming the relevant wavelength range studied and the exposure time secured, if the particular source of a given $M_{\mathrm{UV}}$ has an EW distribution drawn from the sample with $5.5<z<6.3$.

In the case of those sources for which the wavelength range searched does not fully sample the extended $p(z)$, we reduce the detection likelihood by the fraction of the integrated $p(z)$ that lies outside our search range. For each target, we determine its redshift probability function $p(z)$ using the photometric redshift code EAZY (Brammer et al. 2008). To determine the varying visibility function within our search range, we first estimate the noise within an aperture encompassing the expected profile of the line, assuming an emission line width of $10 \AA$ FWHM which is typical of those detected in Paper II. Figure 3 illustrates the $5 \sigma$ EW limit as a function of wavelength (and Ly $\alpha$ redshift) for most sources in our $12 \mathrm{hr}$ LRIS exposure $(6.3<z<7.2)$ and a typical source studied with NIRSPEC $(6.8<z<8.2)$ during a $5 \mathrm{hr}$ exposure. We note that although our survey spans a large range in redshift $(6.3<z<8.8)$, the $p(z)$ distributions for individual galaxies typically span a much smaller range. The average $1 \sigma$ redshift confidence interval for sources in our sample is only $\Delta z=0.43$.

Since the NIRSPEC exposures were usually single-object exposures, the limits vary from source to source depending on the conditions and exposure times. We then apply a completeness correction to account for the fact that an emission line may fall in a noise trough and lie undetected, despite having an intrinsic flux above the $5 \sigma$ limit. To estimate this completeness correction, we follow the methods discussed in detail in Paper II, where we simulate the addition and recovery of fake line emission in our actual spectra, again assuming an FWHM of $10 \AA$. As the absolute limits vary from source to source, our Keck survey is not complete to a fixed EW limit but, provided the limits are well understood for each source, we can readily estimate the probability of seeing $\operatorname{Ly} \alpha$ in our exposures. In the case of the Fontana et al. (2010) FORS2 survey we estimated the night-sky emission from our own LRIS exposures normalizing the limits from numerical data supplied in that paper.

The above simulations can be used to verify that our Keck survey is well placed to search for Ly $\alpha$ emission. Out of the 


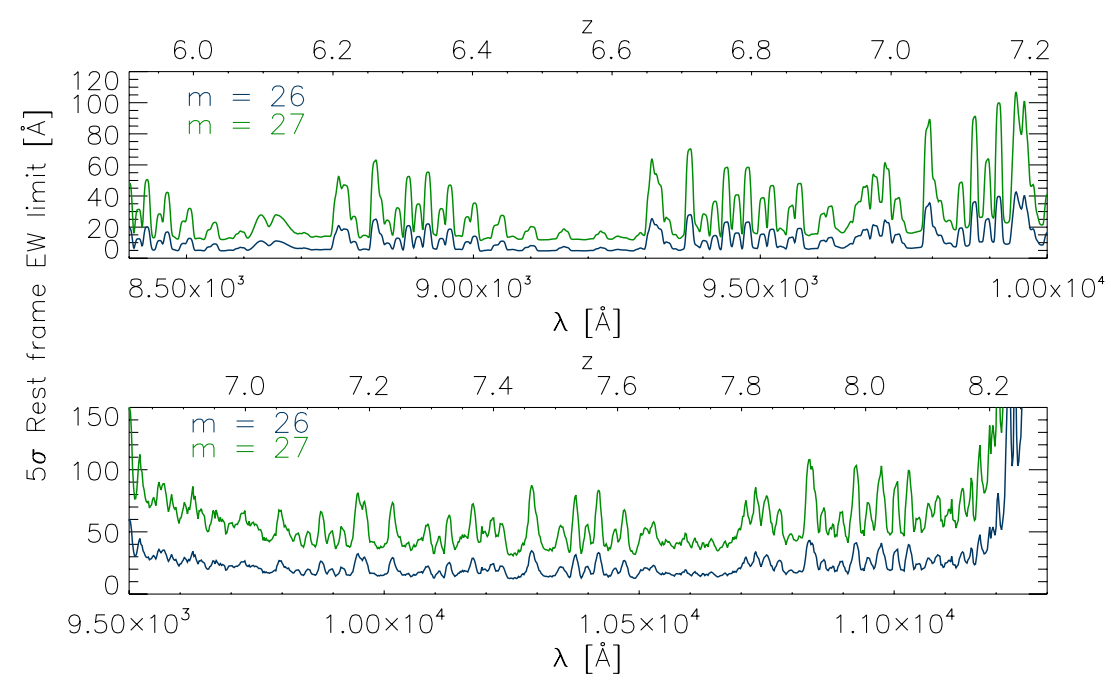

Figure 3. Sensitivity limits to $\mathrm{Ly} \alpha$ emission in our new Keck spectroscopic campaign: the panels show the $5 \sigma$ limiting EW calculated for a typical source studied in our $12 \mathrm{hr}$ multi-slit LRIS exposure (top) and an example $5 \mathrm{hr}$ long slit NIRSPEC observation (bottom). The limits vary from source to source depending on the continuum brightness and the exposure times. An additional completeness correction is taken into account by adding and attempting to recover fake emission lines with fluxes equal to the $5 \sigma$ flux limit at the wavelength of insertion. See the text and Paper II for more detailed discussion.

(A color version of this figure is available in the online journal.)

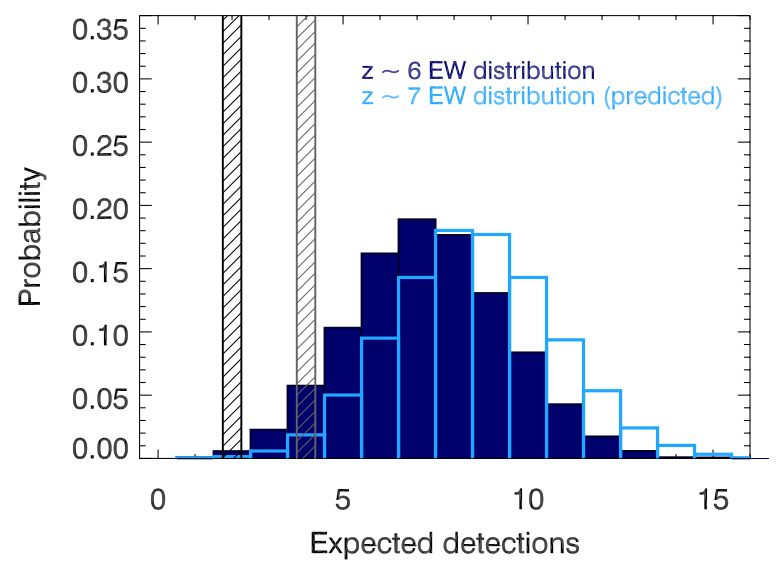

Figure 4. Expected number of detected Ly $\alpha$ emission lines with greater than or equal to $5 \sigma$ significance in the combined Keck and VLT survey of 26 sources. The blue histogram shows the likelihood function for 10,000 Monte Carlo realizations assuming the intrinsic line emission properties follow the luminosity dependence seen in our $5.5<z<6.3 i^{\prime}$-drop sample (Figure 1, top). The open histogram shows the expectation if the fraction of line emitters continues to increase with redshift at the rate described in Paper II. Vertical lines show the recovered number of emitters (robust and maximal including marginal detections in both the Keck and VLT surveys).

(A color version of this figure is available in the online journal.)

combined 26 targets from our survey and that of Fontana et al. (2010), 24 are covered spectroscopically over more than half the integrated probability of their photometric redshift distribution, and 17 are covered over $95 \%$ of the range. Additionally, we are able to determine the fraction of our spectra occulted by $\mathrm{OH}$ sky emission. For example, for a $J=27$ galaxy in one of our LRIS exposures, we are sensitive to lines with $\mathrm{EW} \geqslant 30 \AA$ A over $70 \%$ of our usable spectral range (see Figure 3 ). Similarly, for a $5 \mathrm{hr}$ NIRSPEC exposure of a $J=27$ galaxy, we are sensitive to lines with EW > $55 \AA$ over $49 \%$ our spectral range.

The results of the simulations are shown in Figure 4 where, depending on whether we adopt the EW distribution observed at $5.5<z<6.3$ or that extrapolated to $z \simeq 7$ in Paper II assuming continuity in redshift-dependent increase in line emission seen over $4<z<6$, we would expect to recover 7-8 emission lines in the combined Keck and VLT surveys. In contrast, we have only two robust detections (both in the Keck sample, Figure 2) and at most four including the marginal candidate discussed by Fontana et al. (2010) and HUDF09_1596 at $z=6.905$ shown in Figure 2. Assuming all the targeted sources are at $z>6.3$, given our previously mentioned assumptions, our results reject the input EW distributions at the $99.3 \%$ level of significance (91.4\% if the two marginal detections are included).

We can display the significance of this downturn with increasing redshift in the terms of the fraction of $\operatorname{Ly} \alpha$ emission seen in LBGs, $X(\operatorname{Ly} \alpha)$ as in Paper I. The difficulty we face in creating such a figure is the non-uniform EW limit across the various targets in the Keck and VLT campaigns, in contrast to the more straightforward uniform search we undertook with DEIMOS at $4<z<6$. To account for this, we assume a simple model in which Ly $\alpha$ emission is transmitted without IGM absorption for a fraction $f$ of galaxies, while it is fully extinguished by the IGM for a fraction of galaxies $(1-f)$. We assume that $f=1$ at redshifts below 6 , where the universe is believed to be highly ionized (Fan et al. 2006), and that $f$ is independent of the intrinsic $\mathrm{EW}$ of an Ly $\alpha$ emission line. We caution that an interpretation in terms of absolute values of $f$ is premature, as there is still some debate on whether the IGM is fully ionized at $z \sim 6$ Mesinger (2010), but emphasize that our value of $f$ at $z \sim 7$ is computed relative to the value assumed at $z \sim 6$. Additionally, with the increased fraction of emitters in our $z \sim 6$ sample from Paper II, we do not see any evidence for a decrease in $f$ prior to $z \sim 6$, though we cannot rule it out.

It is important to note that our $f$ is different from $f_{\mathrm{esc}}^{\mathrm{Ly} \alpha}$, commonly defined in the literature as the total escape fraction of Ly $\alpha$ photons (e.g., Hayes et al. 2011). $f_{\text {esc }}^{\text {Ly } \alpha}$ represents the total transmission of $\operatorname{Ly} \alpha$, accounting for both attenuation of photons within the galaxy by mechanisms such as dust, as well as any attenuation by the IGM. Our definition of $f$ is only intended to account for any downturn in the fraction of LBGs which show observable Ly $\alpha$ emission from the $z=6$ (or $z=7$ ) 


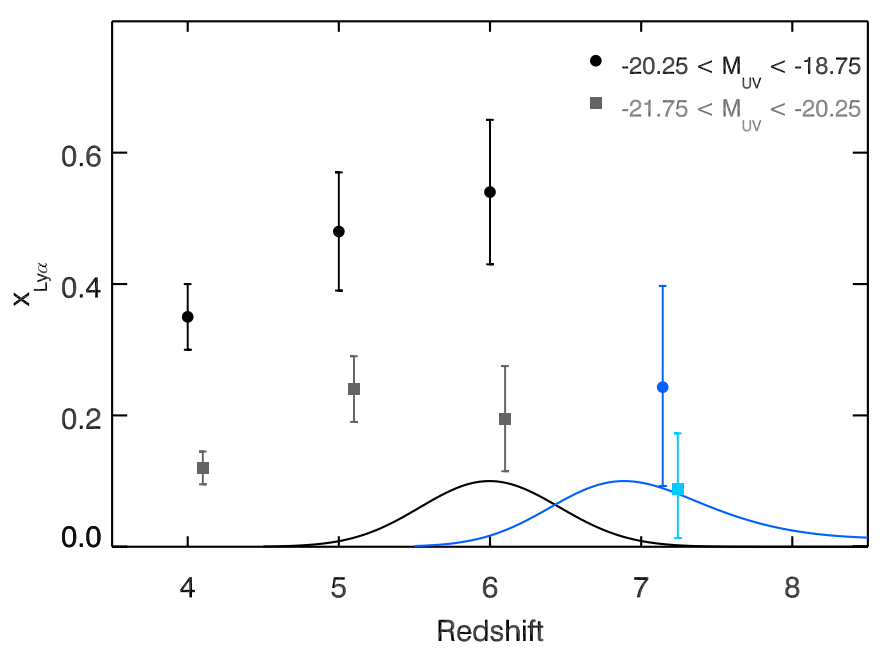

Figure 5. Redshift-dependent fraction of color-selected Lyman break galaxies that reveal $\operatorname{Ly} \alpha$ in emission, $X(\operatorname{Ly} \alpha)$, adjusted as discussed in the text to approximate one within a similar luminosity range with a rest-frame EW in excess of $25 \AA$. Data points for the galaxies with $-21.75<M_{\mathrm{UV}}<-20.25$ are displaced by +0.1 in redshift for clarity. Data over $4<z<6$ is from Paper I and Paper II, and new estimates beyond $z>6.3$ are derived from the present paper, including sources discussed by Fontana et al. (2010). The curves shown represent the aggregate redshift probability distributions for our sources in the $z \simeq 6$ bin (black) and the $z \simeq 7$ bin (blue); probability distributions for individual sources are typically much sharper.

(A color version of this figure is available in the online journal.)

extrapolated EW distributions from Paper II, and represents an IGM extinction averaged over the entire population.

To compute the most likely value of $f$, we undertake Monte Carlo simulations using the previously described EW distributions, but with $f$ now added as a free parameter. We vary $f$ from 0 to 1 in steps of 0.01 , and compute $N=1000$ simulations for each step. We can then calculate the probability distribution for $f$ given our $N_{\text {obs }}=2$ confirmed sources using Bayes' theorem:

$$
p\left(f \mid N_{\mathrm{obs}}=2\right)=\frac{p\left(N_{\mathrm{obs}}=2 \mid f\right) p(f)}{\int_{0}^{1} p\left(N_{\mathrm{obs}}=2 \mid f\right) d f} .
$$

Here, $p(f)$ is the prior probability for $f$, which we take to be uniform for $0 \leqslant f \leqslant 1$, and $p\left(N_{\mathrm{obs}}=2 \mid f\right)$ is the probability, drawn from our Monte Carlo simulations, that we would find $N_{\text {obs }}=2$ sources for a given value of $f$. Assuming that the intrinsic EW distribution for our observed sources is that of Paper II at $z=6$, we find $f=0.45 \pm 0.20$, while using the $z=7$ extrapolated distribution yields $f=0.34_{-0.15}^{+0.24}$. In Figure 5, we plot the value of $X(\operatorname{Ly} \alpha)$ in the same luminosity bins of Paper II, as predicted by our best-fit values of $f$.

We stress that this figure is intended to serve as a continuation of the visualization provided in Papers I and II, rather than a statistical result of our study. Due to our strongly varying limiting EW sensitivity (as a function of both wavelength and object magnitude), choosing a fixed EW limit will exclude a non-negligible fraction of useful data from our analysis. Our Monte Carlo simulations are able to utilize the full data set, simulating whether we would have likely seen a line even when our EW limits are above the fixed thresholds used in Figure 5, and thus represent the major statistical result of this study.

Using the models of McQuinn et al. (2007) to predict what global neutral hydrogen fraction, $X_{\mathrm{HI}_{\mathrm{I}}}$ would be required to account for this decline, we find $X_{\mathrm{H}_{\mathrm{I}}} \simeq 0.44$, and $X_{\mathrm{H}_{\mathrm{I}}} \simeq 0.51$, respectively. The models of Dijkstra et al. (2011), which provide a more comprehensive treatment of $\mathrm{Ly} \alpha$ radiative transfer through outflows, result in an increased value for $X_{\mathrm{H}_{\mathrm{I}}}$ in both cases.

\section{DISCUSSION}

Although we consider the most likely explanation for our observed decrease in the number of LBGs which show observable $\operatorname{Ly} \alpha$ emission to be an increase with redshift in the neutral fraction of the IGM, it is important to remember our assumptions. Foremost we have assumed that all of our 26 targets have true redshifts beyond $z \simeq 6.3$. Should there be low-redshift interlopers or Galactic stars in our new sample, we will overestimate the decline in the Ly $\alpha$ fraction. Second, we have assumed the DEIMOS spectra from Paper II constitute a representative sample for calculating the expected EW distribution for $6.3<z<8.2$. Although the uncertainties here are not as great, we plan further studies with DEIMOS to increase the statistical sample of $5.5<z<6.3$ LBGs.

Of course our observed decrease in the Ly $\alpha$ fraction could also be attributed to an increased opacity arising from dust within the LBGs. However, given the blue UV continuum slopes observed for galaxies with $z>6.3$ (Bouwens et al. 2010a; Dunlop et al. 2011), we consider this explanation unlikely.

Our diagnosis of a possible increase in the neutral hydrogen fraction beyond $z \simeq 6.3$ is supported by the earlier study of Fontana et al. (2010). They found one marginal candidate out of seven targets, whereas we find two robust and one marginal cases out of our 19 targets spanning a larger luminosity and redshift range. Our conclusion is also supported by LAEs studies at $z=5.7$ and 6.5 by Ouchi et al. (2010) and Kashikawa et al. (2011). Compared to $z=5.7$, their LAE sample at $z=6.5$ displays systematically lower EWs for $\mathrm{Ly} \alpha$. They also derive little evolution in the rest UV luminosity function for LAEs, but a decrease in the Ly $\alpha$ luminosity function, which could be explained by an increase in $X_{\mathrm{HI}_{\mathrm{I}}}$. Our derived values of $X_{\mathrm{H}_{\mathrm{I}}}$ are slightly higher than those of Kashikawa et al. (2011), perhaps consistent with our survey probing to higher redshifts than their $z=6.5$ LAEs. Hayes et al. (2011) have recently compiled results from numerous $\mathrm{Ly} \alpha$ and UV luminosity function studies to derive a volumetrically averaged Ly $\alpha$ escape fraction, and find very similar results. Their derived Ly $\alpha$ escape fraction steadily increases with redshift below $z=6$, then tentatively drops off at higher redshifts.

Very recently, Ono et al. (2011) report the convincing detection of $\operatorname{Ly} \alpha$ emission in a small fraction $(3 / 11)$ of LBGs that, by virtue of their selection using Subaru imaging, are more luminous $\left(M_{\mathrm{UV}}<-21\right)$ than most of the objects considered here. Such a complementary campaign targeting luminous LBGs selected from larger volumes will provide further insight into whether reionization is responsible for the declining fraction of line emission.

We note that our measured decrease in the fraction of LBGs with strong $\operatorname{Ly} \alpha$ potentially agrees with the result of Cowie et al. (2011). Although they argue against any evidence for reionization at $z=6.5$, they find that $\sim 24 \%$ of galaxies at this redshift show strong Ly $\alpha$ emission, comparable to the fraction we detect in this work, spread across a larger redshift range.

With the new generation of multi-object, near-infrared spectrographs, such as MOSFIRE, set to come online soon, the prospects for this field are bright. In addition to the significant multiplexing advantage, the increased sensitivity of these detectors will allow us to probe the lower luminosity ranges at $z \geqslant 6.5$ to $\mathrm{EW}$ limits comparable to those in 
Paper II between sky lines. Having such a statistical sample is key for allowing the quantification of any change in the hydrogen neutral fraction.

We thank the referee for valuable comments which improved the manuscript, George Becker for allowing us to use his NIRSPEC reduction pipeline, and Mark Dijkstra for his helpful discussion. R.S.E. and M.A.S. thank the Institute of Astronomy, Cambridge, where this work was completed, for its support. We also recognize and acknowledge the very significant cultural role and reverence that the summit of Mauna Kea has always had within the indigenous Hawaiian community. We are most fortunate to have the opportunity to conduct observations from this mountain. D.P.S. acknowledges financial support from a postdoctoral fellowship from the Science Technology and Research Council. B.E.R. is supported by a Hubble Fellowship grant, program number HST-HF-51262.01-A provided by NASA from the Space Telescope Science Institute, which is operated by the Association of Universities for Research in Astronomy, Incorporated, under NASA contract NAS5-26555. J.S.D. acknowledges the support of the Royal Society via a Wolfson Research Merit award, and the support of the European Research Council via an Advanced Grant. R.J.M. acknowledges the support of the Royal Society via a University Research Fellowship.

\section{REFERENCES}

Brammer, G. B., van Dokkum, P. G., \& Coppi, P. 2008, ApJ, 686, 1503 Bouwens, R. J., Illingworth, G. D., Oesch, P. A., et al. 2010a, ApJ, 708, L69

Bouwens, R. J., Illingworth, G. D., Oesch, P. A., et al. 2010b, ApJ, 709, L133

Bradley, L. D., Bouwens, R. J., Zitrin, A., et al. 2011, ApJ, submited (arXiv:1104.2035)

Bunker, A., Wilkins, S., Ellis, R. S., et al. 2010, MNRAS, 409, 855B

Cowie, L. L., Hu, E. M., \& Songaila, A. 2011, ApJ, 735, L38

Dijkstra, M., Mesinger, A., \& Wyithe, J. S. B. 2011, MNRAS, 414, 2139
Dunlop, J. S., McLure, R. J., Robertson, B. E., et al. 2011, MNRAS, submitted (arXiv:1102.5065)

Faber, S. M., Phillips, A. C., Kibrick, R. I., et al. 2003, Proc. SPIE, 4841, 1657

Fan, X., Strauss, M. A., Becker, R. H., et al. 2006, AJ, 132, 117

Finkelstein, S. L., Papovich, C., Giavalisco, M., et al. 2010, ApJ, 719, 1250

Fontana, A., Vanzella, E., Pentericci, L., et al. 2010, ApJ, 725, L205

Grogin, D., Kocevski, D. D., Faber, S. M., et al. 2011, ApJS, submitted (arXiv:1105.3753)

Hathi, N. P., Ryan, R. E., Jr., Cohen, S. H., et al. 2010, ApJ, 720, 1708

Hayes, M., Schaerer, D., Östlin, G., et al. 2011, ApJ, 730, 8

Kashikawa, N., Shimasaku, K., Matsuda, Y., et al. 2011, ApJ, 734, 119

Kelson, D. D. 2003, PASP, 115, 1688

Koekemoer, A. M., Faber, S. M., Ferguson, H. C., et al. 2011, ApJS, submitted (arXiv:1105.3754)

Malhotra, S., \& Rhoads, J. E. 2004, ApJ, 617, L5

McLean, I. S., Becklin, E. E., Bendiksen, O., et al. 1998, Proc. SPIE, 3354, 566

McLure, R. J., Dunlop, J. S., Cirasuolo, M., et al. 2010, MNRAS, 403, 960

McLure, R. J., Dunlop, J. S., de Ravel, L., et al. 2011, MNRAS, in press (arXiv:1102.4881)

McQuinn, M., Hernquist, L., Zaldarriaga, M., \& Dutta, S. 2007, MNRAS, 381, 75

Mesinger, A. 2010, MNRAS, 407, 1328

Oke, J. B., Cohen, J. G., Carr, M., et al. 1995, PASP, 107, 375

Oke, J. B., \& Gunn, J. E. 1983, ApJ, 266, 713

Ono, Y., Ouchi, M., Mobasher, B., et al. 2011, ApJ, submitted (arXiv:1107.3159)

Ouchi, M., Shimasaku, K., Furusawa, H., et al. 2010, ApJ, 723, 8690

Pentericci, L., Fontana, A., Vanzella, E., et al. 2011, ApJ, submitted (arXiv:1107.1376)

Postman, M., Coe, D., Benitez, N., et al. 2011, arXiv:1106.3328

Richard, J., Smith, G. P., Kneib, J.-P., et al. 2010, MNRAS, 404, 325

Richard, J., Stark, D. P., Ellis, R. S., et al. 2008, ApJ, 685, 705

Robertson, B. E., Ellis, R. S., Dunlop, J. S., McLure, R. J., \& Stark, D. P. 2010, Nature, 468, 49

Stark, D. P., Ellis, R. S., Chiu, K., Ouchi, M., \& Bunker, A. 2010, MNRAS, 408, 1628 (Paper I)

Stark, D. P., Ellis, R. S., \& Ouchi, M. 2011, ApJ, 728, L2 (Paper II)

Trenti, M., Bradley, L. D., Stiavelli, M., et al. 2011, ApJ, 727L, 39T

Vanzella, E., Giavalisco, M., Dickinson, M., et al. 2009, ApJ, 695, 1163

Vanzella, E., Pentericci, L., Fontana, A., et al. 2011, ApJ, 730, L35

Wilkins, S. M., Bunker, A. J., Ellis, R. S., et al. 2010, MNRAS, 403, 938

Wilkins, S. M., Bunker, A. J., Lorenzoni, S., \& Caruana, J. 2011, MNRAS, 411, 23

Yan, H., Yan, L., Zamojski, M. A., et al. 2011, ApJ, 728, L22 